\title{
Research Article \\ Viewing Sea Level by a One-Dimensional Random Function with Long Memory
}

\author{
Ming Li, ${ }^{1}$ Carlo Cattani, ${ }^{2}$ and Seng-Yong Chen ${ }^{3}$ \\ ${ }^{1}$ School of Information Science \& Technology, East China Normal University, Shanghai 200241, China \\ ${ }^{2}$ Department of Pharmaceutical Sciences (DiFarma), University of Salerno, Via Ponte Don Melillo, \\ 84084 Fisciano, SA, Italy \\ ${ }^{3}$ College of Computer Science, Zhejiang University of Technology, Hangzhou 310023, China
}

Correspondence should be addressed to Ming Li, ming_lihk@yahoo.com

Received 12 September 2010; Accepted 5 October 2010

Academic Editor: Cristian Toma

Copyright (C) 2011 Ming Li et al. This is an open access article distributed under the Creative Commons Attribution License, which permits unrestricted use, distribution, and reproduction in any medium, provided the original work is properly cited.

\begin{abstract}
Sea level fluctuation gains increasing interests in several fields, such as geoscience and ocean dynamics. Recently, the long-range dependence (LRD) or long memory, which is measured by the Hurst parameter, denoted by $H$, of sea level was reported by Barbosa et al. (2006). However, reports regarding the local roughness of sea level, which is characterized by fractal dimension, denoted by $D$, of sea level, are rarely seen. Note that a common model describing a random function with LRD is fractional Gaussian noise (fGn), which is the increment process of fractional Brownian motion (fBm) (Beran (1994)). If using the model of $\mathrm{fGn}, \mathrm{D}$ of a random function is greater than 1 and less than 2 because $D$ is restricted by $H$ with the restriction $D=2-H$. In this paper, we introduce the concept of one-dimensional random functions with LRD based on a specific class of processes called the Cauchy-class (CC) process, towards separately characterizing the local roughness and the long-range persistence of sea level. In order to achieve this goal, we present the power spectrum density (PSD) function of the CC process in the closed form. The case study for modeling real data of sea level collected by the National Data Buoy Center (NDBC) at six stations in the Florida and Eastern Gulf of Mexico demonstrates that the sea level may be one-dimensional but LRD. The case study also implies that the CC process might be a possible model of sea level. In addition to these, this paper also exhibits the yearly multiscale phenomenon of sea level.
\end{abstract}

\section{Introduction}

Although, in general, a secular change trend of relative mean sea level over a wide range of time scale in one year, or 10 years, or 100 years, and a broad range of space scale, such as global scale, is certainly a focus in the aspect of ocean dynamics, see, for example, [1, 2], Lyard et al. [3], local fluctuations of sea level, or sea level dynamics at small-time scales, such as daily or hourly, are essential for some practical issues, such as navigations, coastal 
engineering, military debarkation, and tide power production, see, for example, Liu [4], [5, Chapter 8], Wyrtki and Nakahara [6].

Recently, Barbosa et al. [7] reported their work to exhibit that North Atlantic sea level has the property of LRD. They analyzed the LRD behavior of the sea level based on a commonly used asymptotic PSD expression of LRD random functions for $f \rightarrow 0$, that is, the PSD of $1 / f$ noise, where $f$ is frequency. However, they neither gave the analytic expression of sea level in the closed form nor mentioned the local roughness of sea level. The aim of this paper is to address our research in the aspect of fluctuations of sea level, towards making a considerable step further with the contributions in the following three folds.

(i) We bring in the concept of LRD but one-dimensional random functions. This concept is introduced based on the Cauchy-class (CC) process. The autocorrelation function (ACF) of the CC process can be seen in the field of geostatistics [8], but its PSD in the closed form is a problem unsolved. We will present a solution to that problem in this paper.

(ii) In the aspect of fractal analysis of sea level, we will propose two new results. One is that the sea level may be one-dimensional though LRD, quantitatively characterizing the local roughness of sea level. The other is the Hurst parameter, $H$, of sea level is time varying, exhibiting its multiscale property.

(iii) We will exhibit that the CC model well fits in with the sea level in the Florida and Eastern Gulf of Mexico accurately. By accurately, we mean that the mean-square error (MSE) between the PSD of the CC process and the measured PSD is in the order of magnitude of $10^{-3}$ or less.

Note that LRD time series can be considered in the class of fractal time series, see, for example, Beran [9], Mandelbrot [10]. The most commonly used LRD model is the fractional Gaussian noise (fGn) introduced by Mandelbrot [11], where the fractal dimension $D$ of $f G n$, which measures the local roughness of fGn, linearly relates to its $H$ by $D=2-H[9,10]$. In this paper, we separately characterize $D$ and $H$ of sea level. More precisely, with the CC model, $D$ of sea level keeps the constant one while $H$ varies from 0.5 to 1 .

In the rest of the paper, we present the closed form of the PSD of the CC process in Section 2. Section 3 demonstrates the results of data modeling. Discussions are arranged in Section 4. Finally, Section 5 concludes the paper.

\section{CC Process: A One-Dimensional Random Function with LRD}

In this section, we explain the CC process, which is a one-dimensional random function with LRD. The aim of this section is to present the closed form of the PSD of the CC process with LRD.

\subsection{Brief of LRD Processes}

Let $X(t)$ be a stationary process with mean zero for $-\infty<t<\infty$. Let $R(\tau)=\mathrm{E}[X(t) X(t+\tau)]$ be the autocorrelation function (ACF) of $X(t)$, where $\tau$ designates the time lag. Then, if $R(\tau)$ is nonintegrable, $X(t)$ is of LRD while it is of short-range dependence (SRD) if $R(\tau)$ is integrable. For the power-law type ACF that has the asymptotic property given by

$$
R(\tau) \sim c \tau^{-\beta}, \quad(t \longrightarrow 0),
$$


where $c>0$ is a constant, one has the LRD condition expressed by $0<\beta<1[9,10]$. The parameter $\beta$ is the index of LRD. Expressing $\beta$ by the Hurst parameter $H$ so that $\beta=2-2 H$ yields the LRD condition $0.5<H<1$. The larger the $H$ value, the stronger the long-range persistence.

Denote $S(\omega)$ the Fourier transform (FT) of $R(\tau)$. Then, near origin, $S(\omega)$ has the asymptotic property expressed by

$$
S(\omega) \sim c_{1} \omega^{-(1+\beta)} \quad(\omega \longrightarrow 0)
$$

which implies power-law type PSD, where $c_{1}>0$ depends on $c$ and $\beta$.

\subsection{Process}

\subsubsection{ACF of CC Process}

In this research, we focus on the ACF discussed in the geostatistics. It is given by (Chilès and Delfiner [8, page 86])

$$
C(\tau)=\left(1+\frac{|\tau|^{2}}{a^{2}}\right)^{-b_{1}}, \quad \tau \in \mathbb{R}, a>0, b_{1}>0 .
$$

We call a Gaussian process $X(t)$ that follows (2.3) the CC process.

The above ACF is obviously regular for $\tau \approx 0$. Since one can replace $|\tau| / a$ by $|\tau|$, we simplify the above by the following:

$$
C(\tau)=\left(1+|\tau|^{2}\right)^{-b_{1}}, \quad \tau \in \mathbb{R}, b_{1}>0
$$

For facilitating the discussion of LRD, we rewrite the above by

$$
C(\tau)=\left(1+|\tau|^{2}\right)^{-b / 2}, \quad \tau \in \mathbb{R}, b>0 .
$$

Equation (2.5) reduces to the ordinary Cauchy model when $b=2$.

\subsubsection{LRD Condition of CC Process}

It is obviously seen that the LRD condition of the CC process is $0<b<1$ because $C(\tau) \sim$ $\tau^{-b}(\tau \rightarrow \infty)$. The SRD condition is $b>1$. The Hurst parameter of the CC process is computed by

$$
H=1-\frac{b}{2}
$$




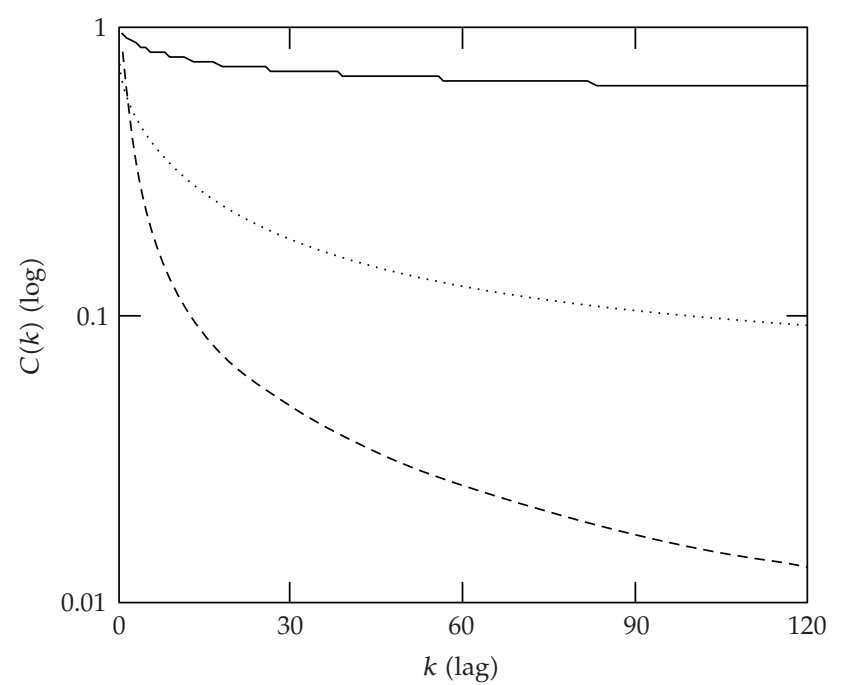

Figure 1: ACF of CC process. Solid line: ACF for $H=0.95$, dot line: ACF for $H=0.75$, dash line: ACF for $H=0.55$.

For facilitating the illustration of $C(\tau)$ in terms of $H$, we write $C(\tau)$ by

$$
C(\tau, H)=\left(1+|\tau|^{2}\right)^{H-1}
$$

Figure 1 indicates $C(\tau, H)$ for three values of $H$.

\subsubsection{PSD of CC Process}

Because $C(\tau)$ is nonintegrable for $0<b<1$, the FT of $C(\tau)$ does not exist in the domain of ordinary functions if $0<b<1$. This reminds us that the PSD of the CC process with LRD should be treated as a generalized function over the Schwartz space of test functions.

Note that the FT of $C(\tau)$ expressed by (2.5) remains unknown, to our best knowledge. By computing the FT of $C(\tau)$ expressed by (2.5) in the domain of generalized functions (see Gelfand and Vilenkin [12]), we obtain

$$
S(\omega)=\int_{-\infty}^{\infty}\left(1+|\tau|^{2}\right)^{-b / 2} e^{-j \omega \tau} d \tau=\frac{2^{(1-b) / 2}}{\sqrt{\pi} \Gamma(b / 2)}|\omega|^{1 / 2(b-1)} K_{1 / 2(b-1)}(|\omega|),
$$

where $K_{v}(\cdot)$ is the modified Bessel function of the second kind (Olver [13, page 254]), which is expressed by

$$
K_{v}(z)=\frac{\Gamma(v+1 / 2)(2 z)^{v}}{\sqrt{\pi}} \int_{0}^{\infty} \frac{\cos t d t}{\left(t^{2}+z^{2}\right)^{v+1 / 2}}
$$


The function $S(\omega)$ has the following asymptotic properties. When $|z|$ is small, see [13, (8.12), page 252], one has

$$
K_{v}(z) \sim \frac{1}{2} \Gamma(v)\left(\frac{1}{2} z\right)^{-v}, \text { for } z \longrightarrow 0^{+}, v>0
$$

Therefore,

$$
S(\omega) \sim \frac{\Gamma[(1 / 2)(1-b)]}{2^{b} \sqrt{\pi \Gamma(b / 2)}}|\omega|^{b-1} \quad(|\omega| \longrightarrow 0)
$$

The above expression exhibits that $S(\omega) \rightarrow \infty$ if $|\omega| \rightarrow 0$ when $0<b<1$. This is the LRD condition described in the frequency domain, implying that the CC process with LRD is a kind of $1 / f$ noise.

The PSD of the CC model with LRD has a singularity at $\omega=0$. However, we may regularize it so that the regularized PSD is finite at $\omega=0$. Denote $S_{0}(\omega)$ the regularized PSD when $0<b<1$. Then,

$$
S_{0}(\omega)=\frac{S(\omega)}{\lim _{\omega \rightarrow 0} S(\omega)}
$$

In this case, $\lim _{\omega \rightarrow 0} S_{0}(\omega)=1$. In what follows, the PSD is assumed to be the regularized one unless otherwise stated. Figure 2 illustrates the regularized PSD of the CC process with LRD.

\subsubsection{Fractal Dimension of CC Process}

Following the work by Adler [14], Hall and Roy [15], and Kent and Wood [16], one can obtain the expression (2.13) if $C(\tau)$ is sufficiently smooth on $(0, \infty)$ and if

$$
C(0)-C(\tau) \sim c|\tau|^{\alpha}, \quad \text { for }|\tau| \longrightarrow 0,
$$

where $c$ is a constant, then, the fractal dimension is given by

$$
D=2-\frac{\alpha}{2}
$$

Taking into account (2.14) and $\alpha=2$ in (2.5), we immediately obtain the fractal dimension of the CC process, which is given by

$$
D=1
$$

because for (2.5), we have

$$
C(0)-C(\tau) \sim|\tau|^{2}, \quad \text { for }|\tau| \longrightarrow 0 \text {. }
$$




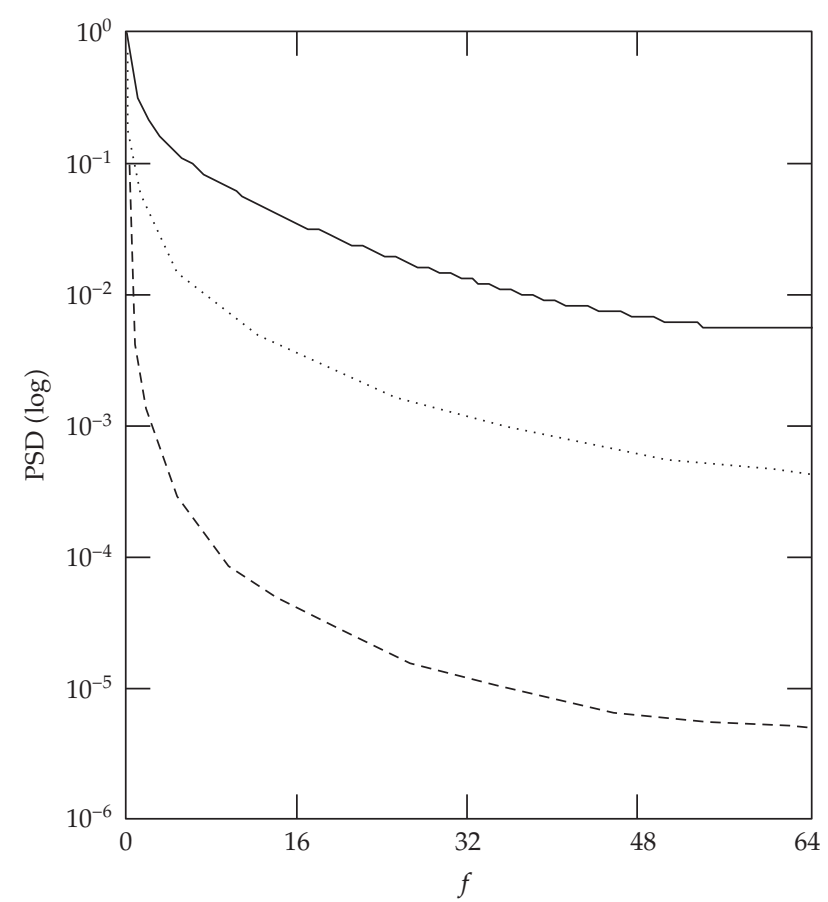

Figure 2: Regularized PSD. Solid line: PSD for $H=0.95$, dot line: PSD for $H=0.75$, dash line: PSD for $H=$ 0.55 .

\section{Case Study in Sea Level}

\subsection{Data}

NDBC, being a part of the US National Weather Service (NWS) [17], provides immense data for the scientific research, ranging from air temperature to sea level. We use the data collected at six stations named LONF1, LKWF1, SAUF1, SMKUF1, SPGF1, and VENF1, respectively. They are located in the Florida and Eastern Gulf of Mexico, see [18].

The data are in the category of Water Level accessible from [19]. All data were hourly recorded with ten devices denoted by TGn $(n=01,02, \ldots, 10)$. Without losing generality, the following uses the data from the device TG01. Denote the data series by $x_{\_}$__yyyy $(t)$, where $\mathrm{s}$ is the name of the measurement station and yyyy stands for the index of year. Denote $x \_$s_yyyy $(t)$ and $S_{\_}$s_yyyy $(f)$ as the measured time series and the measured PSD at the

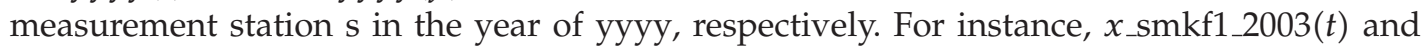
$S \_s m k f 1$ 2003 $(f)$ represent the measured time series and the measured PSD at the station SMKF1 in 2003, respectively.

The data that are labeled 99 are regarded as outliers or missing ones and they are replaced by the mean of that series. According to the suggestion from NDBC, $10 \mathrm{ft}$ was subtracted from every value of $x_{\_}$s_yyyy $_{(}(t)$ before estimating its PSD.

Practically, a spectrum is measured on a block-by-block basis (Mitra and Kaiser [20], Li [21]). Therefore, there are errors (e.g., truncation error) in spectrum measurement. To reduce errors, the spectrum is usually measured by averaging spectral estimates of blocks of data. Let $B$ be the block size and let $M$ be the average count, respectively. We sectioned the data in the nonoverlapping case. $M$ is selected such that $0<[L-(B \times M)]<B$, where $L$ is the total 
Table 1: Measured data at LONF1.

\begin{tabular}{|c|c|c|c|c|}
\hline Series name & Record date and time & $L$ & $B$ & $M$ \\
\hline$x \_l o n f 11 \_1998(t)$ & 0:00, 3 Nov.-23:00, 31 Dec. 1998 & 1416 & 256 & 5 \\
\hline$x \_l o n f 1 \_1999(t)$ & 0:00, 1 Jan.-21:00, 31 Dec. 1999 & 8757 & 256 & 34 \\
\hline$x \_$lonf1_2000(t) & 0:00, 1 Jan.-23:00, 31 Dec. 2000 & 8484 & 256 & 33 \\
\hline$x$ lonf1_2001 $(t)$ & 0:00, 1 Jan.-23:00, 31 Dec. 2001 & 8760 & 256 & 34 \\
\hline$x \_$lonf1_2002(t) & 0:00, 1 Jan.-23:00, 31 Dec. 2002 & 8760 & 256 & 34 \\
\hline$x \_$lonf1_2003(t) & 0:00, 1 Jan.-23:00, 31 Dec. 2003 & 8697 & 256 & 33 \\
\hline$x \_$lonf1_2004 $(t)$ & 0:00, 1 Jan.-23:00, 31 Dec. 2004 & 8758 & 256 & 34 \\
\hline$x$ _lonf1_2005(t) & 0:00, 1 Jan.-23:00, 31 Dec. 2005 & 8750 & 256 & 34 \\
\hline
\end{tabular}

Table 2: Measured data at LKWF1.

\begin{tabular}{|c|c|c|c|c|}
\hline Series name & Record date and time & $L$ & $B$ & $M$ \\
\hline$x \_l \mathrm{kwf1} 1996(t)$ & 0:00, 1 Jan.-23:00, 31 Dec. 1996 & 8208 & 256 & 32 \\
\hline$x \_$lkwf1_1997(t) & 0:00, 1 Jan.-23:00, 31 Dec. 1997 & 7776 & 256 & 30 \\
\hline$x \_$lkwf1_1998(t) & 0:00, 1 Jan.-23:00, 31 Dec. 1998 & 8736 & 256 & 34 \\
\hline$x \_\mathrm{kwf1} 1$ 1999(t) & 0:00, 1 Jan.-23:00, 31 Dec. 1999 & 8760 & 256 & 34 \\
\hline$x \_\mathrm{kwf} 12000(t)$ & 0:00, 1 Jan.-17:00, 26 Feb. 2000 & 1362 & 256 & 5 \\
\hline$x \_\mathrm{lkwf1} 2001(t)$ & 17:00, 8 Aug.-23:00, 31 Dec. 2001 & 2972 & 256 & 11 \\
\hline$x \_l k w f 1 \_2002(t)$ & 0:00, 1 Jan.-23:00, 31 Dec. 2002 & 8740 & 256 & 34 \\
\hline$x \_l \mathrm{kwf} 1 \_2003(t)$ & 0:00, 1 Jan.-23:00, 31 Dec. 2003 & 8582 & 256 & 33 \\
\hline$x \_l \mathrm{kwf1} 12004(t)$ & 0:00, 1 Jan.-14:00, 5 Oct. 2004 & 6655 & 256 & 25 \\
\hline
\end{tabular}

length of $x_{-}$s_yyyy $(t)$. Tables $1,2,3,4,5$, and 6 list the measured data and the settings for the spectrum measurements.

\subsection{Fitting the Data of PSD and H Estimations}

The key parameter for characterizing the LRD of the sea level is $H$. The literature regarding $H$ estimation is affluent. Commonly used estimators of $H$ are $R / S$ analysis, maximum likelihood method, variogram-based methods, box-counting, detrended fluctuation analysis, spectrum regression, correlation regression, see, for example, [10, 11], Peng et al. [22], Kantelhardt et al. [23], Taqqu et al. [24], and Yin et al. [25]. In this paper, we use the method of spectrum regression to estimate $H$.

After obtaining a measured PSD $S \_$s_yyyy $(f)$, we do the data fitting with the theoretic PSD $S_{0}(f)$ of the CC process by using the least-square fitting. Denote the cost function by

$$
J(b)=\frac{2}{B} \sum_{k}\left[S_{0}(f)-S_{-} s_{-} \text {yyyy }(f)\right]^{2},
$$

where $S_{-}$s_yyyy $(f)$ is in the normalized case. The derivative of $J$ with respect to $b$, which will be zero when $J$ is minimum, yields $b_{0}$ or equivalently $H_{0}$, which is the solution of $d J / d b=0$.

Figure 3 indicates 4 series at the station LONF1. Each starts from the first data point to the 256 th one, that is, about the first 10 days of data. The data fitting between the 
Table 3: Measured data at SAUF1.

\begin{tabular}{lcccc}
\hline Series name & Record date and time & $L$ & $B$ & $M$ \\
\hline x_sauf1_1996(t) & 0:00, 1 Jan.-14:00, 10 Aug. 1996 & 5511 & 256 & 21 \\
x_sauf1_1997 $(t)$ & 0:00, 25 Feb.-23:00, 31 Dec. 1997 & 6240 & 256 & 24 \\
x_sauf1_1998(t) & 0:00, 1 Jan. -23:00, 31 Dec. 1998 & 8736 & 256 & 34 \\
x_sauf1_1999 $(t)$ & 0:00, 1 Jan. -23:00, 31 Dec. 1999 & 8136 & 256 & 31 \\
x_sauf1_2000(t) & 0:00, 1 Jan. -23:00, 31 Dec. 2000 & 8715 & 256 & 34 \\
x_sauf1_2001 $(t)$ & 0:00, 1 Jan. -21:00, 31 Dec. 2001 & 8758 & 256 & 34 \\
x_sauf1_2002 $(t)$ & 20:00, 6 Feb. -23:00, 20 Aug. 2002 & 4684 & 256 & 18 \\
\hline
\end{tabular}

Table 4: Measured data at SMKF1.

\begin{tabular}{lcccc}
\hline Series name & Record date and time & $L$ & $B$ & $M$ \\
\hline x_smkf1_1998(t) & 0:00, 3 Nov.-23:00, 31 Dec. 1998 & 1416 & 256 & 5 \\
x_smkf1_1999(t) & 0:00, 1 Jan.-23:00, 31 Dec. 1999 & 7775 & 256 & 30 \\
x_smkf1_2000(t) & 0:00, 1 Aug.-23:00, 31 Dec. 2000 & 3542 & 256 & 13 \\
x_smkf1_2001(t) & 0:00, 1 Jan.-23:00, 31 Dec. 2001 & 5776 & 256 & 22 \\
x_smkf1_2002(t) & 0:00, 1 Jan.-23:00, 31 Dec. 2002 & 8742 & 256 & 34 \\
x_smkf1_2003(t) & 0:00, 1 Jan.-23:00, 31 Dec. 2003 & 5851 & 256 & 22 \\
x_smkf1_2004(t) & 0:00, 1 Jan.-23:00, 31 Dec. 2004 & 8439 & 256 & 32 \\
x_smkf1_2005(t) & 0:00, 1 Jan.-23:00, 31 Dec. 2005 & 8667 & 256 & 33 \\
\hline
\end{tabular}

measured PSD and the theoretical one for each series is demonstrated in Figure 4 . By the least square fitting, we have the estimated $H$ values $0.973,0.975,0.991,0.990$ for $x \_l o n f 1 \_1998(t)$, $x \_l o n f 1 \_1999(t), x \_l o n f 1 \_2002(t), x \_l o n f 1 \_2005$, respectively (Table 7). The MSE for the data fitting of each series is in the order of magnitude of $10^{-5}$ (Table 7). Estimates of $H$ for other series are summarized in Tables 7, 8, 9, 10, 11, and 12 .

\subsection{Summarized Results of H Estimation}

See Tables 7-12.

\section{Discussions}

It is worth noting that the real data of sea level at several sites may not be enough to infer that the discussed CC model provides us with a general pattern of sea level. However, considering that the MSEs of the curve fitting for all measured series being in the order of magnitude of $10^{-4}$ or less, see Tables $7-12$, the CC process might yet be useful for studying sea level modeling as well as fluctuations of sea level at both large-time scales and small-time ones. This research suggests that sea level may be one-dimensional as (2.15) implied, which is a quantitative description of the local roughness of sea level.

Judging from the results in Tables 7-12, we see that sea level is LRD since all values of $H$ s are greater than 0.5 . However, $H$ varies yearly, see Tables $7-12$. Hence, sea level is multiscaled. Considering the Taqqu's theorem for the relationship between LRD and heavytailed probability density (see Samorodnitsky and Taqqu [26], Abry et al. [27]), we infer that the sea level is heavy-tailed. Therefore, as a side product, the present results support the point 
Table 5: Measured data at SPGF1.

\begin{tabular}{lcccc}
\hline Series name & Record date and time & $L$ & $B$ & $M$ \\
\hline x_spgf1_1996(t) & 0:00, 1 Jan.-23:00, 15 Dec. 1996 & 8616 & 256 & 33 \\
x_spg1_1997( $t$ ( $)$ & 0:00, 6 Mar.-23:00, 15 Dec. 1997 & 7080 & 256 & 27 \\
x_spg1_1998 $(t)$ & 0:00, 1 Jan.-23:00, 7 Jan. 1998 & 168 & 32 & 5 \\
\hline
\end{tabular}

Table 6: Measured data at VENF1.

\begin{tabular}{lcccc}
\hline Series name & Record date and time & $L$ & $B$ & $M$ \\
\hline$x_{\text {_venf1_2002 }(t)}$ & 0:00, 1 Oct.-23:00, 31 Dec. 2002 & 2208 & 256 & 8 \\
$x_{\text {_ven1_2003 }(t)}$ & 0:00, 1 Jan.-23:00, 31 Dec. 2003 & 8760 & 256 & 34 \\
$x_{-}$ven1_2004 $(t)$ & 0:00, 1 Jan.-16:00, 7 Jan. 2004 & 634 & 256 & 2 \\
\hline
\end{tabular}

Table 7: $H$ estimates at LONF1.

\begin{tabular}{|c|c|c|c|}
\hline Series name & $b_{0}$ & $H_{0}$ & MSE \\
\hline$x$ lonf11_1998(t) & 0.054 & 0.973 & $7.944 \times 10^{-5}$ \\
\hline$x \_l o n f 1 \_1999(t)$ & 0.050 & 0.975 & $6.919 \times 10^{-5}$ \\
\hline$x$ lonf1 $2000(t)$ & 0.280 & 0.986 & $2.107 \times 10^{-5}$ \\
\hline$x$ lonf1_2001 $(t)$ & 0.021 & 0.990 & $1.390 \times 10^{-5}$ \\
\hline$x \_$lonf1_2002(t) & 0.018 & 0.991 & $1.156 \times 10^{-5}$ \\
\hline$x$ lonf1_2003 $(t)$ & 0.019 & 0.991 & $1.253 \times 10^{-5}$ \\
\hline$x \_$lonf1_2004 $(t)$ & 0.017 & 0.991 & $1.241 \times 10^{-5}$ \\
\hline$x \_$lonf1_2005(t) & 0.020 & 0.990 & $1.462 \times 10^{-5}$ \\
\hline
\end{tabular}

Table 8: $H$ estimates at LKWF1.

\begin{tabular}{|c|c|c|c|}
\hline Series name & $b_{0}$ & $H_{0}$ & MSE \\
\hline$x$ x_lkwf1_1996(t) & 0.055 & 0.973 & $2.234 \times 10^{-4}$ \\
\hline$x \_l k w f 1 \_1997(t)$ & 0.060 & 0.970 & $2.174 \times 10^{-4}$ \\
\hline$x \_l k w f 1 \_1998(t)$ & 0.055 & 0.973 & $2.150 \times 10^{-4}$ \\
\hline$x \_l k w f 1 \_1999(t)$ & 0.056 & 0.972 & $2.140 \times 10^{-4}$ \\
\hline$x \_$lkwf1_2000(t) & 0.065 & 0.967 & $2.385 \times 10^{-4}$ \\
\hline$x \_l$ kwf1_2001(t) & 0.060 & 0.970 & $1.725 \times 10^{-4}$ \\
\hline$x \_\mathrm{kwff1} 2002(t)$ & 0.045 & 0.977 & $1.814 \times 10^{-4}$ \\
\hline$x \_\mathrm{kwf} 1 \_2003(t)$ & 0.043 & 0.978 & $3.144 \times 10^{-4}$ \\
\hline$x \_l \mathrm{kwf} 1 \_2004(t)$ & 0.040 & 0.980 & $1.789 \times 10^{-4}$ \\
\hline
\end{tabular}

Table 9: $H$ estimates at SAUF1.

\begin{tabular}{|c|c|c|c|}
\hline Series name & $b_{0}$ & $H_{0}$ & MSE \\
\hline$x_{\text {x_sauf1_1996(t) }}$ & 0.130 & 0.935 & $7.606 \times 10^{-4}$ \\
\hline$x \_$sauf1_1997(t) & 0.100 & 0.950 & $5.376 \times 10^{-4}$ \\
\hline$x \_$sauf1_1998(t) & 0.100 & 0.950 & $5.614 \times 10^{-4}$ \\
\hline$x_{\text {_ssauf1_1999 }}(t)$ & 0.150 & 0.925 & $8.320 \times 10^{-4}$ \\
\hline$x \_$sauf1_2000(t) & 0.085 & 0.985 & $4.797 \times 10^{-4}$ \\
\hline$x \_$sauf1_2001(t) & 0.082 & 0.959 & $4.697 \times 10^{-4}$ \\
\hline$x_{\text {_ssauf1_2002 }}(t)$ & 0.075 & 0.962 & $4.070 \times 10^{-4}$ \\
\hline
\end{tabular}




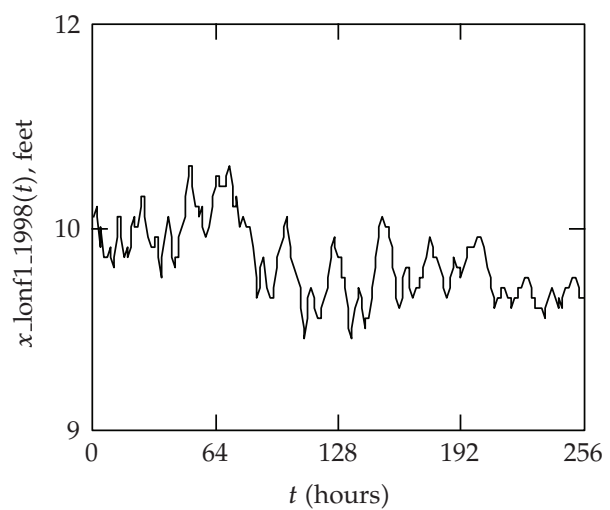

(a)

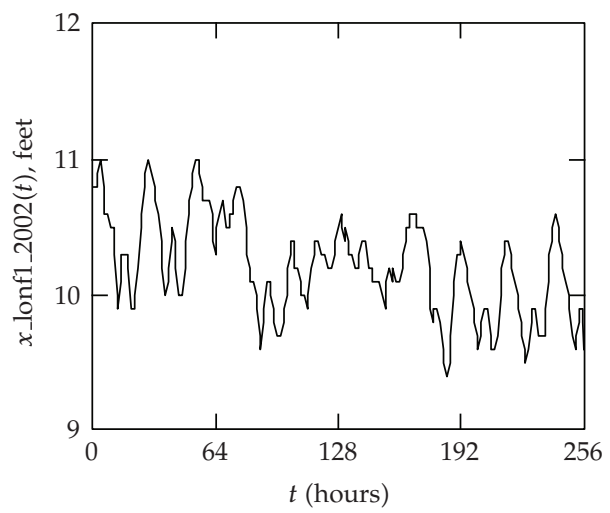

(c)

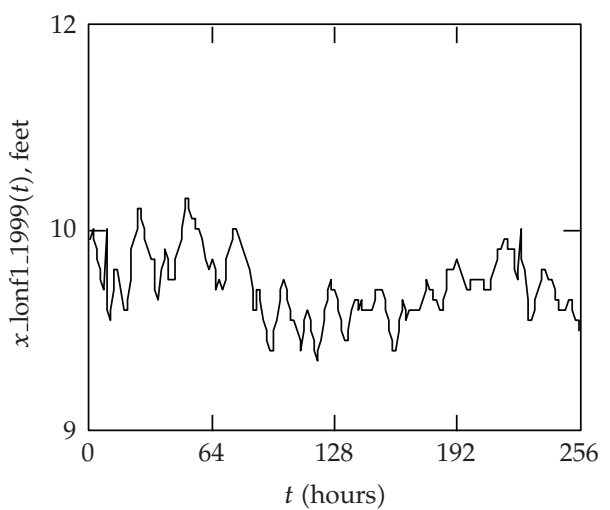

(b)

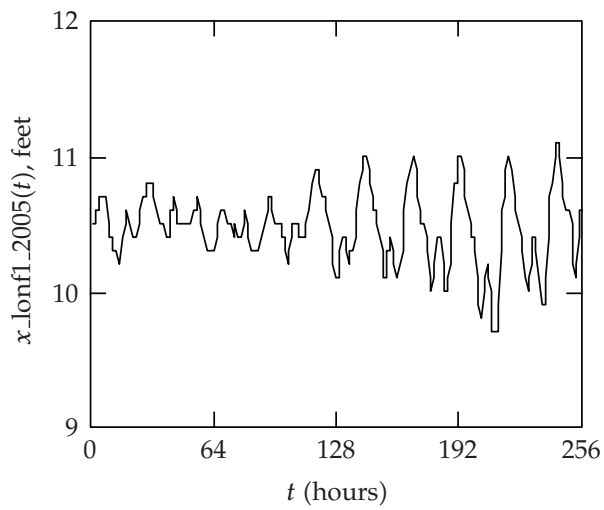

(d)

Figure 3: Data series at LONF1. (a) $x \_l o n f 1 \_1998(t)$. (b) $x \_l o n f 1 \_1999(t)$. (c) $x \_l o n f 1 \_2002(t)$. (d) $x$ lonf1_2005(t).

Table 10: $H$ estimates at SMKF1.

\begin{tabular}{|c|c|c|c|}
\hline Series name & $b_{0}$ & $H_{0}$ & MSE \\
\hline$x \_s m k f 1 \_1998(t)$ & 0.035 & 0.983 & $9.632 \times 10^{-5}$ \\
\hline$x \_$smkf1_1999(t) & 0.045 & 0.977 & $8.555 \times 10^{-5}$ \\
\hline$x \_s m k f 1 \_2000(t)$ & 0.045 & 0.977 & $7.955 \times 10^{-5}$ \\
\hline$x \_$smkf1_2001(t) & 0.035 & 0.983 & $7.963 \times 10^{-5}$ \\
\hline$x \_$smkf1_2002(t) & 0.025 & 0.987 & $7.369 \times 10^{-5}$ \\
\hline$x \_s m k f 1 \_2003(t)$ & 0.022 & 0.989 & $7.322 \times 10^{-5}$ \\
\hline$x \_$smkf1_2004(t) & 0.019 & 0.991 & $7.268 \times 10^{-5}$ \\
\hline$x \_s m k f 1 \_2005(t)$ & 0.019 & 0.989 & $6.910 \times 10^{-5}$ \\
\hline
\end{tabular}

Table 11: $H$ estimates at SPGF1.

\begin{tabular}{lccc}
\hline Series name & $b_{0}$ & $H_{0}$ & MSE \\
\hline$x \_$spgf1_1996(t) & 0.045 & 0.977 & $2.217 \times 10^{-4}$ \\
$x \_s p g f 1 \_1997(t)$ & 0.055 & 0.973 & $2.158 \times 10^{-4}$ \\
$x \_$spgf1_1998(t) & 0.085 & 0.960 & $9.074 \times 10^{-4}$ \\
\hline
\end{tabular}




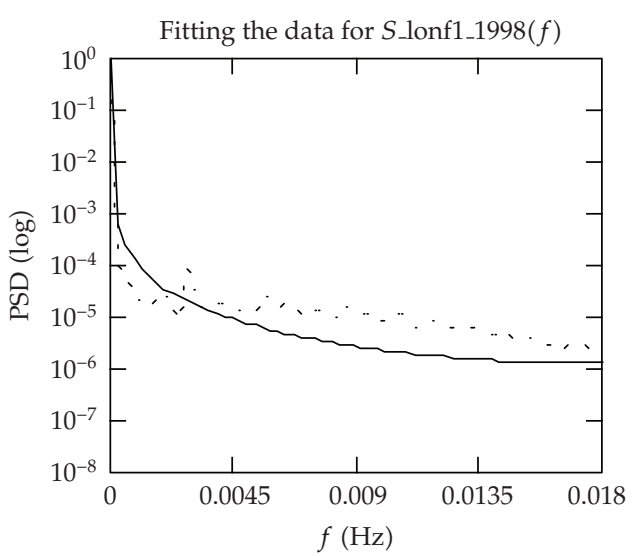

(a)

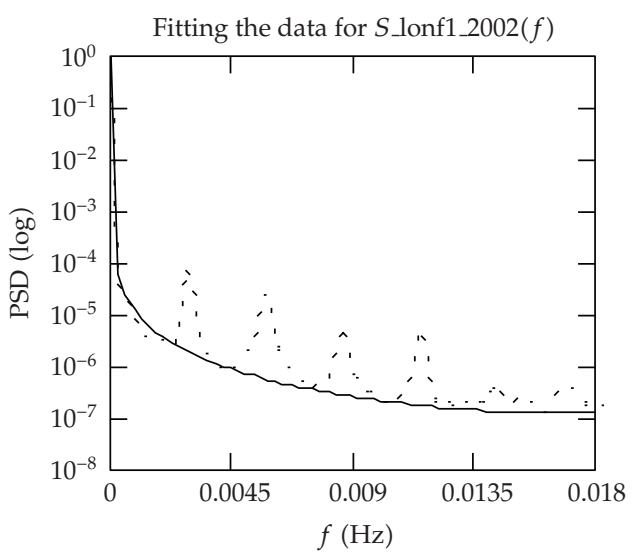

(c)

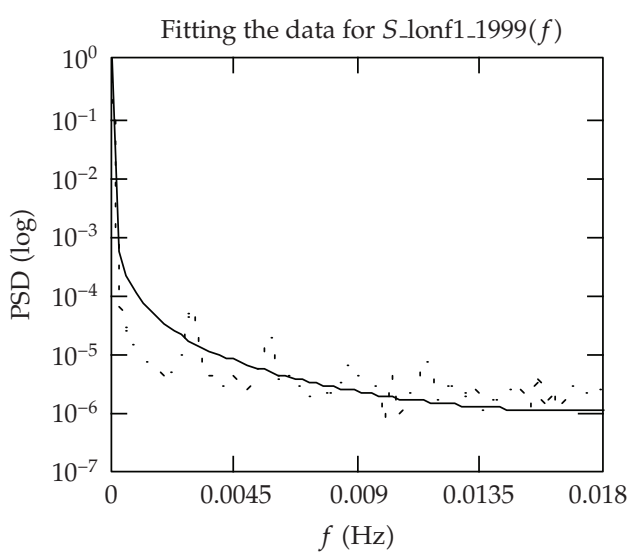

(b)

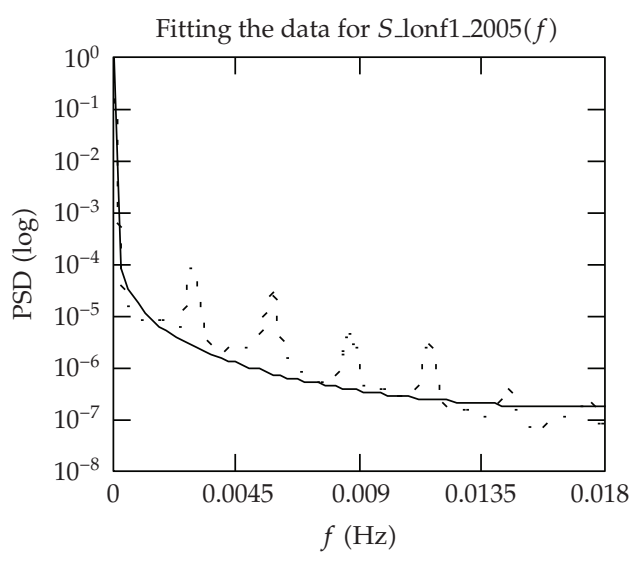

(d)

Figure 4: Fitting the data of PSD at LONF1. Dot line: measured PSD, solid line: theoretical PSD. (a) Fitting the data for S_lonf1_1998 ( $f$ ). (b) Fitting the data for S_lonf1_1999 $(f)$. (c) Fitting the data for S_lonf1_2002( $f$ ). (d) Fitting the data for S_lonf1_2005 $(f)$.

Table 12: $H$ estimates at VENF1.

\begin{tabular}{llll}
\hline Series name & $b_{0}$ & $H_{0}$ & MSE \\
\hline$x_{-}$venf1_2002 $(t)$ & 0.042 & 0.979 & $8.091 \times 10^{-5}$ \\
$x_{-}$venf1_2003 $(t)$ & 0.039 & 0.981 & $8.147 \times 10^{-5}$ \\
$x_{-}$venf1_2004 $(t)$ & 0.030 & 0.985 & $1.204 \times 10^{-4}$ \\
\hline
\end{tabular}

of view that heavy-tailed distributions, equivalently LRD, play a role in the field of disaster analysis in geoscience as Pisarenko and Rodkin noted [28].

Note the selection of the test data used in this research is arbitrary, only for the purpose of demonstrating the application case of the CC process to the dynamics of sea level. This paper may be a beginner to investigate dynamics of sea level using a type of one-dimensional random functions with LRD. Other methods [29-41], such as wavelets and short-term pulses may be helpful for the research in this regard. 


\section{Conclusions}

We have presented the closed form of the PSD of the CC process. We have explained that this class of processes is one dimensional but LRD. Applying it to modeling the sea level in the Florida and Eastern Gulf of Mexico implies a suggestion that the discussed CC process might be a candidate in sea level modeling.

\section{Acknowledgments}

This work was supported in part by the National Natural Science Foundation of China under project Grant no.s, 60573125, 60873264, 61070214, 60870002, and 70871077, the 973 plan under project no. 2011CB302800, NCET, and the Science and Technology Department of Zhejiang Province (2009C21008, 2010R10006, 2010C33095, Y1090592). The National Data Buoy Center is highly appreciated.

\section{References}

[1] Geophysics Study Committee, National Research Council, Sea-Level Change, National Academic Press, 1990.

[2] Committee on Engineering Implications of Changes in Relative Mean Sea Level, Marine Board, National Research Council, Responding to Changes in Sea Level: Engineering Implications, National Academic Press, 1987.

[3] F. Lyard, F. Lefevre, T. Letellier, and O. Francis, "Modelling the global ocean tides: modern insights from FES2004," Ocean Dynamics, vol. 56, no. 5-6, pp. 394-415, 2006.

[4] G.-Y. Liu, Ocean Geography, The Press of Beijing Normal University, 1989.

[5] Ocean School of Ningbo, Ed., Oceanography, Ocean Press, 1986.

[6] K. Wyrtki and S. Nakahara, "Monthly maps of sea level anomalies in the Pacific 1975-1981," Tech. Rep. JIMAR 84-0085, HIG-843, Joint Institute of Marine and Atmospheric Research and Hawaii Institute of Geophysics, University of Hawaii, Honolulu, Hawaii, USA, August 1984.

[7] S. M. Barbosa, M. J. Fernandes, and M. E. Silva, "Long-range dependence in North Atlantic sea level," Physica A, vol. 371, no. 2, pp. 725-731, 2006.

[8] J.-P. Chilès and P. Delfiner, Geostatistics, Modeling Spatial Uncertainty, Wiley Series in Probability and Statistics: Applied Probability and Statistics, John Wiley \& Sons, New York, NY, USA, 1999.

[9] J. Beran, Statistics for Long-Memory Processes, vol. 61 of Monographs on Statistics and Applied Probability, Chapman and Hall, New York, NY, USA, 1994.

[10] B. B. Mandelbrot, Gaussian Self-Affinity and Fractals, Springer, New York, NY, USA, 2001.

[11] B. B. Mandelbrot and J. W. Van Ness, "Fractional Brownian motions, fractional noises and applications," SIAM Review, vol. 10, no. 4, pp. 422-437, 1968.

[12] I. M. Gelfand and K. Vilenkin, Generalized Functions, Vol. 1, Academic Press, New York, NY, USA, 1964.

[13] F. W. J. Olver, Asymptotics and Special Functions, Academic Press, New York, NY, USA, 1974.

[14] R. J. Adler, The Geometry of Random Fields, John Wiley \& Sons, Chichester, UK, 1981.

[15] P. Hall and R. Roy, "On the relationship between fractal dimension and fractal index for stationary stochastic processes," The Annals of Applied Probability, vol. 4, no. 1, pp. 241-253, 1994.

[16] J. T. Kent and A. T. A. Wood, "Estimating the fractal dimension of a locally self-similar Gaussian process by using increments," Journal of the Royal Statistical Society B, vol. 59, no. 3, pp. 679-699, 1997.

[17] http://www.nws.noaa.gov/.

[18] http://seaboard.ndbc.noaa.gov/maps/Florida.shtml.

[19] http://seaboard.ndbc.noaa.gov/historical_data.shtml.

[20] S. K. Mitra and J. F. Kaiser, Handbook for Digital Signal Processing, John Wiley \& Sons, New York, NY, USA, 1993. 
[21] M. Li, "A method for requiring block size for spectrum measurement of ocean surface waves," IEEE Transactions on Instrumentation and Measurement, vol. 55, no. 6, pp. 2207-2215, 2006.

[22] C.-K. Peng, S. V. Buldyrev, S. Havlin, M. Simons, H. E. Stanley, and A. L. Goldberger, "Mosaic organization of DNA nucleotides," Physical Review E, vol. 49, no. 2, pp. 1685-1689, 1994.

[23] J. W. Kantelhardt, E. Koscielny-Bunde, H. H. A. Rego, S. Havlin, and A. Bunde, “Detecting long-range correlations with detrended fluctuation analysis," Physica A, vol. 295, no. 3-4, pp. 441-454, 2001.

[24] M. S. Taqqu, V. Teverovsky, and W. Willinger, "Estimators for long-range dependence: an empirical study," Fractals, vol. 3, no. 4, pp. 785-798, 1995.

[25] X.-A. Yin, X.-H. Yang, and Z.-F. Yang, “Using the R/S method to determine the periodicity of time series," Chaos, Solitons and Fractals, vol. 39, no. 2, pp. 731-745, 2009.

[26] G. Samorodnitsky and M. S. Taqqu, Stable Non-Gaussian Random Processes, Stochastic Models with Infinite Variance, Stochastic Modeling, Chapman \& Hall, New York, NY, USA, 1994.

[27] P. Abry, P. Borgnat, F. Ricciato, A. Scherrer, and D. Veitch, "Revisiting an old friend: on the observability of the relation between long range dependence and heavy tail," Telecommunication Systems, vol. 43, no. 3-4, pp. 147-165, 2010.

[28] V. Pisarenko and M. Rodkin, Heavy-Tailed Distributions in Disaster Analysis, vol. 30, Springer, 2010.

[29] C. Cattani, "Harmonic wavelet approximation of random, fractal and high frequency signals," Telecommunication Systems, vol. 43, no. 3-4, pp. 207-217, 2010.

[30] C. Cattani, "Fractals and hidden symmetries in DNA," Mathematical Problems in Engineering, vol. 2010, Article ID 507056, 22 pages, 2010.

[31] C. Cattani, "Shannon wavelets for the solution of integrodifferential equations," Mathematical Problems in Engineering, vol. 2010, Article ID 408418, 22 pages, 2010.

[32] M. Li and S. C. Lim, "Modeling network traffic using generalized Cauchy process," Physica A, vol. 387, no. 11, pp. 2584-2594, 2008.

[33] M. Li and W. Zhao, "Representation of a stochastic traffic bound," IEEE Transactions on Parallel and Distributed Systems, vol. 21, no. 9, pp. 1368-1372, 2010.

[34] E. G. Bakhoum and C. Toma, "Dynamical aspects of macroscopic and quantum transitions due to coherence function and time series events," Mathematical Problems in Engineering, vol. 2010, Article ID 428903, 13 pages, 2010.

[35] E. G. Bakhoum and C. Toma, "Relativistic short range phenomena and space-time aspects of pulse measurements," Mathematical Problems in Engineering, vol. 2008, Article ID 410156, 20 pages, 2008

[36] S. Y. Chen, Y. F. Li, and J. Zhang, "Vision processing for realtime 3-D data acquisition based on coded structured light," IEEE Transactions on Image Processing, vol. 17, no. 2, pp. 167-176, 2008.

[37] S. Y. Chen, Y. F. Li, Q. Guan, and G. Xiao, "Real-time three-dimensional surface measurement by color encoded light projection," Applied Physics Letters, vol. 89, no. 11, Article ID 111108, 2006.

[38] S. He and F. Liu, "Robust $L_{2}-L_{\infty}$ filtering of time-delay jump systems with respect to the finite-time interval," Mathematical Problems in Engineering, vol. 2011, Article ID 839648, 2011.

[39] W. B. Mikhael and T. Yang, "A gradient-based optimum block adaptation ICA technique for interference suppression in highly dynamic communication channels," EURASIP Journal on Applied Signal Processing, vol. 2006, Article ID 84057, 10 pages, 2006.

[40] T.-Y. Sung, Y.-S. Shieh, and H.-C. Hsin, “An efficient VLSI linear array for DCT/IDCT using subband decomposition algorithm," Mathematical Problems in Engineering, vol. 2010, Article ID 185398, 21 pages, 2010.

[41] D. She and X. Yang, "A new adaptive local linear prediction method and its application in hydrological time series," Mathematical Problems in Engineering, vol. 2010, Article ID 205438, 15 pages, 2010. 


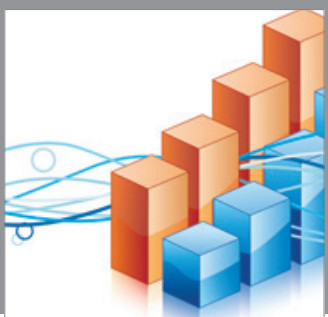

Advances in

Operations Research

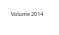

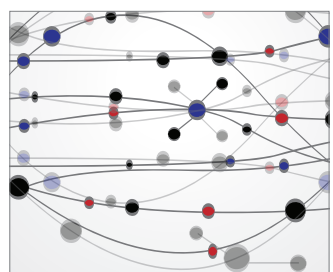

\section{The Scientific} World Journal
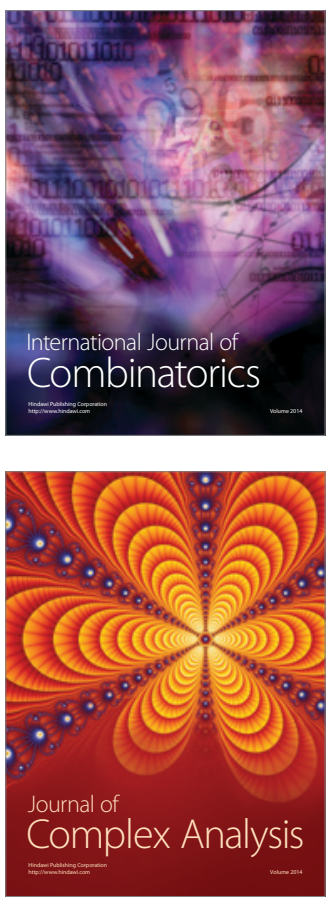

International Journal of

Mathematics and

Mathematical

Sciences
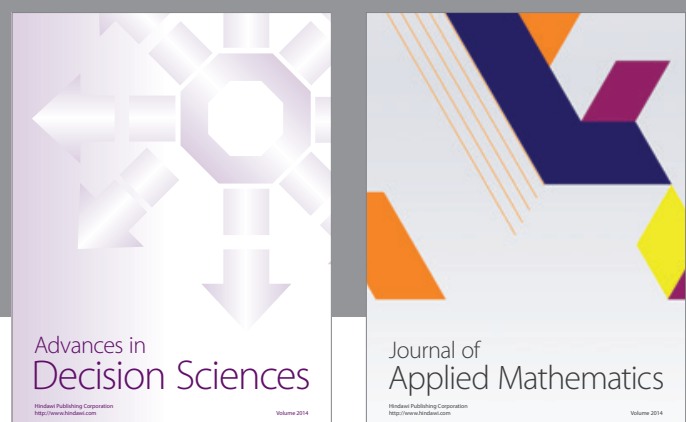

Journal of

Applied Mathematics
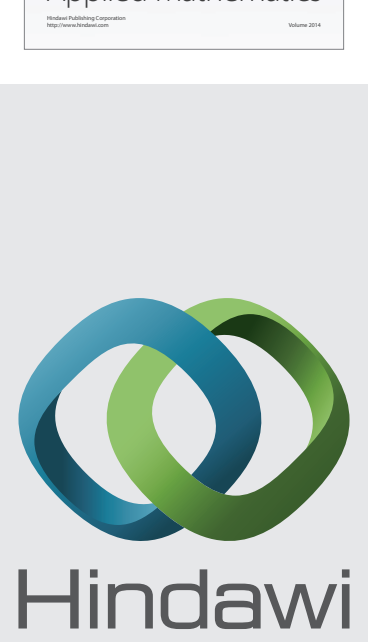

Submit your manuscripts at http://www.hindawi.com
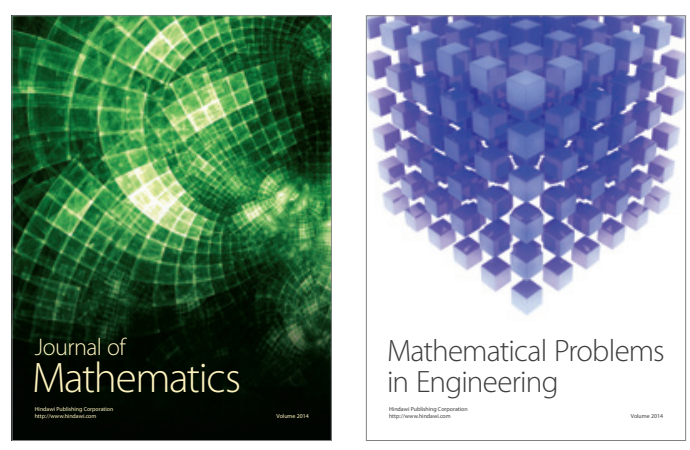

Mathematical Problems in Engineering
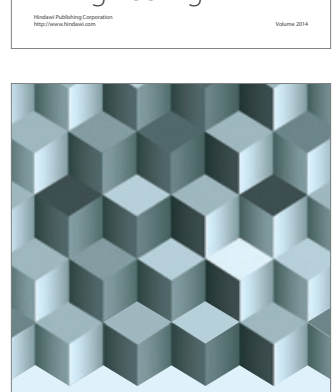

Journal of

Function Spaces
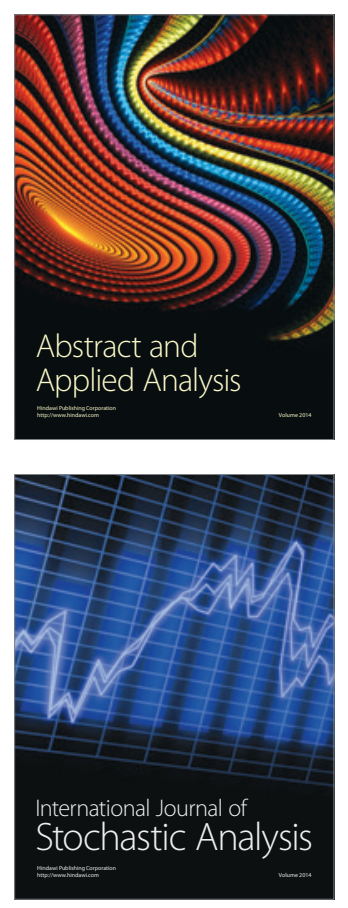

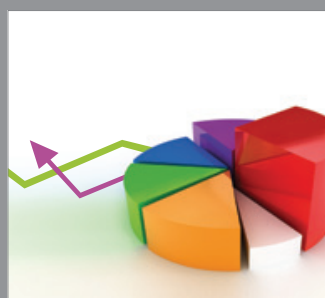

ournal of

Probability and Statistics

Promensencen
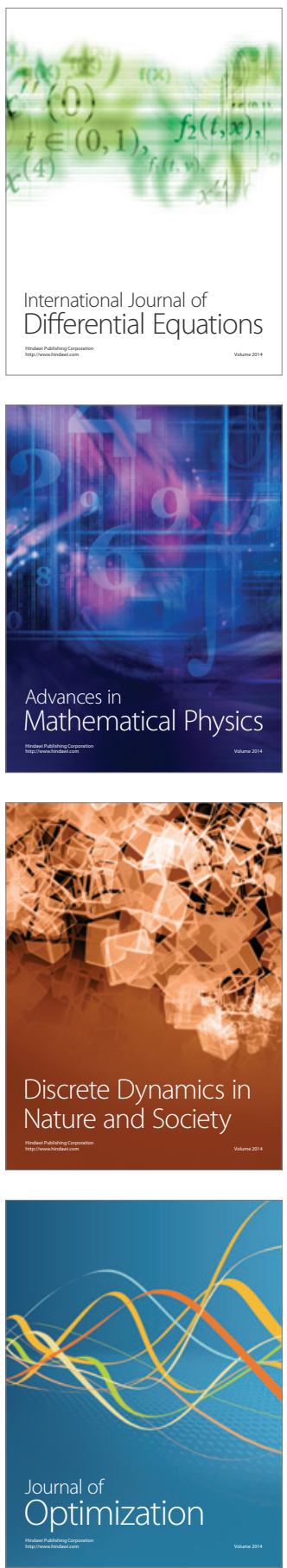